\title{
Effect of Au thickness on Microfabricated Strain Gauge for the Application of Flexible Polymer Heart Valve Membrane
}

Yuli Lin ${ }^{1}$, Kuan-Tung $\mathrm{Su}^{1}$, Gin-Shin Chen ${ }^{2}$, Jia-Shing Liu ${ }^{2}$, Chih-Cheng Lu ${ }^{2}$ and

Ned H.C. Hwang ${ }^{2}$

${ }^{1}$ Department of Mechanical Engineering,Chung Hua University, Hsinchu, 300 Taiwan ROC

${ }^{2}$ Division of Medical Engineering Research, National Health Research Institutes,Zhunan, Taiwan

Flexible polymer heart valves have been expected to be potential heart valve prostheses to replace diseased and malfunctioned heart valves [1]. However, the membrane of flexible polymer heart valve is prone to fatigue fracture, which hinders its practicality and application [2]. Numerical simulation was often utilized to investigate the stress-strain relation when the flexible polymer heart valve was operated [3]. While, accurate prediction of stress-strain relation is difficult to obtain when numerical simulation method was used. Therefore, it is necessary to directly investigate the strain distribution in the membrane when it exposed to a dynamic flow, so as to improve the structure of flexible polymer heart valve.

In this study, a novel concept regarding microfabricated strain gauge embedded in the membrane of flexible polymer heart valve for in situ measurement the strain distribution is introduced. This microfabricated strain gauge should be designed such that interference in the characteristic and motion of polymer heart valve due to its small size and merged into the membrane could be avoid as much as possible. The overall size of designed strain gauge is $1 \mathrm{~mm} \times 1 \mathrm{~mm}$. The gold thin film was deposited onto the polyurethane (PU) membrane utilizing sputtering process. Various thickness of Au coating was deposited on PU substrate to investigate the variation of resistivity. The geometry of strain gauge was manufactured by dry etching process using ProMaster ArF 193nm excimer-laser. The thickness of Au coating was measured by Surfcorder ET4000. The microfabricated strain gauge was observed by scanning electron microscopy. These designed strain gauges will be embedded on the surface of membrane to form a Rosette to measure plain strain of a small area. The set of strain gauges will also be arranged at stress-concentrated locations obtained by finite element simulation.

Fig. 1(a) and (b) shows the geometry of a designed strain gauge demonstrating that a well patterned strain gauge on the PU substrate with $100 \mu \mathrm{m}$ in thickness is obtained. It should be noted that well setting of the excimer-laser is crucial in microfabricating this strain gauge. It was also found that the resistivity of the designed strain gauge was decrease as increasing the thickness of Au coating. The 
maximum resistivity of $4.4 \times 10^{-5} \Omega$-cm can be produced at strain gauge with $20 \mathrm{~nm}$ in thickness. Fig. 2 depicts the distribution of resistivity as changing the thickness of Au film.

[1] T.G.Mackay et.al., Biomaterials 17(1996) 1857-1863.

[2] G.B.Bernacca et. al., Bimaterials 16(1995) 279-285.

[3] Q.Lin et. al., Int. J. Computer Application in Technology, Vol.21, Nos. 1/2, 2004.

[4] This research was supported by NSC94-2216-E-216-009, CHU94-2216-E-216-009 and CHU94-TR-09.
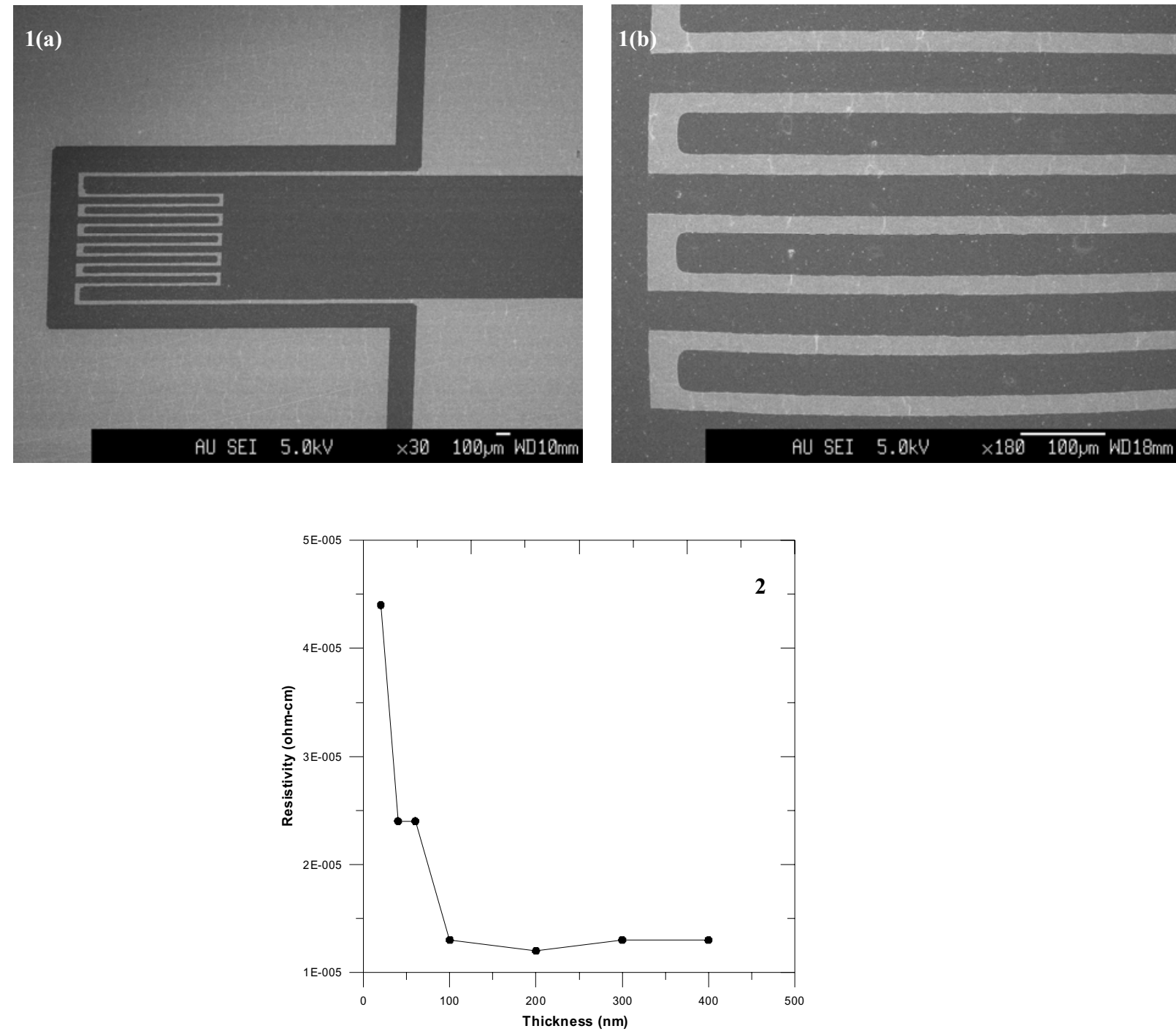

Figure 1. (a) The designed strain gauge of Au thin film.

(b) The designed strain gauge of Au thin film in higher magnification from (a)

Figure 2. Distribution of resistivity as changing the thickness of Au thin film. 